\title{
COVID-19 vaccination with BNT162b2 and ChAdOx1 vaccines induces nasal neutralizing antibodies
}

Philippe Gevaert ${ }^{1}$, Declercq Jozefien ${ }^{2}$, Tobback Els ${ }^{3}$, Stijn Vanhee ${ }^{2}$, Natalie Deruyck ${ }^{1}$, Gerlo Sarah ${ }^{4}$, and Vandekerckhove Linos ${ }^{4}$

${ }^{1}$ Universiteit Gent

${ }^{2}$ Vlaams Instituut voor Biotechnologie

${ }^{3}$ Universitair Ziekenhuis Gent

${ }^{4}$ Universiteit Gent Vakgroep Inwendige Ziekten

July 17,2021

COVID-19 vaccination with BNT162b2 and ChAdOx1 vaccines induces nasal neutralizing antibodies

Declercq Jozefien ${ }^{(1,2,5)^{*}}$, Tobback Els ${ }^{(3)^{*}}$, Vanhee Stijn ${ }^{(2,5)}$, Natalie De Ruyck ${ }^{(1)}$, Gerlo Sarah $^{(4)}$, Gevaert Philippe $^{(1)^{* *}}$, Vandekerckhove Linos ${ }^{(3,4)^{* *}}$

*shared co-first authorship

** shared last authorship

1. Upper Airways Research Lab URL, Department of Otorhinolaryngology, Ghent University, Ghent, Belgium

2. Laboratory of immunoregulation, VIB Center for Inflammation Research, Ghent, Belgium

3. Department of General Internal Medicine, Ghent University Hospital, Ghent, Belgium

4. HIV Cure Research Centre, Department of Internal Medicine and Pediatrics, Ghent University, Ghent, Belgium

5. Department of Internal Medicine and Pediatrics, Ghent University, Ghent, Belgium

Corresponding author:

Prof. Dr. Philippe Gevaert

Upper Airways Research Lab URL, Department of Otorhinolaryngology, Ghent University,

C Heymanslaan 10, 1P1

Ghent, Belgium

$+3293324922$

Philippe.gevaert@ugent.be

Financial support: no funding

Word count: 591

To the editor, 
to date, several vaccines against severe acute respiratory coronavirus 2 (SARS-CoV-2) have proven to effectively reduce severe illness $(1,2)$. To promote herd immunity and reduce virus circulation, vaccines need to effectively reduce transmission risk. The nasal cavity is the first entrance point for SARS-CoV-2 and it has been suggested that viral replication is most efficient in the upper airways (3). Local neutralizing antibodies (NAbs) in the nasal mucosa can play an important role in preventing SARS-CoV-2 infection and transmission by limiting viral replication and shedding. While induction of systemic neutralizing humoral responses has been shown for both natural infection and upon vaccination with BNT162b2 and ChAdOx1, the presence of NAbs in the nasal mucosa upon vaccination remains unclear. A recent not peer reviewed report described the potential of BNT162b2 to induce NAbs in the nasal cavity, but did not consider prior COVID-19 as a potentiator of vaccine-induced local immune responses (4). Local humoral responses after vaccination with viral vector-based vaccines, another type of frequently used SARS-CoV-2 vaccines, has not been investigated. As the induction of nasal NAbs might be a key marker of vaccine efficacy, we set out to determine whether SARS-CoV-2 mRNA (BNT162b2) and viral vector based (ChAdOx1) vaccines can induce local neutralizing responses in the nasal cavity.

To address this question, we collected serum and nasal secretions from subjects visiting the COVID-19 vaccination center at the University Hospital Ghent, Belgium. Sampling took place just before the first SARS-CoV-2 vaccination, with either BNT162b2 or ChAdOx1, and at 13-40 days after the second dose with the same vaccine. All patients provided written informed consent. Collection of nasal secretions was done as described previously (5). In all samples, SARS-CoV-2 NAbs were determined by using the Elabscience SARS-CoV-2 Neutralization Antibody ELISA Kit. NAbs levels expressed as inhibition rates were determined, with an inhibition [?] $20 \%$ considered positive and $<20 \%$ considered negative. Forty-six subjects, mainly females, were included in the study (Table 1). Half of these subjects had a history of prior COVID-19. Prior to vaccination, 16 subjects had NAbs in serum and 4 in nasal secretions. At second sampling, except for one, all subjects showed NAbs in their serum, regardless of the vaccine received (Figure $1 \mathrm{~A}+\mathrm{B})$. In nasal secretions, NAbs were observed in the majority of subjects $(\mathrm{n}=23 ; 96 \%)$ vaccinated with BNT162b2 and in about half of the subjects $(\mathrm{n}=13 ; 59 \%)$ vaccinated with ChAdOx1 at second sampling $(\mathrm{p}=0.0032$; Fisher's exact test) (Figure 1C). Moreover, the ACE2 binding inhibition in nasal secretion was higher in subjects vaccinated with BNT162b2 compared to those vaccinated with ChAdOx1 ( $<<0.0001 ; 2$-way repeated-measures ANOVA with Sidak's multiple comparisons test) (Figure 1D). Induction of NAbs occurred irrespective of prior SARS-CoV-2 infection or the presence of patient reported allergy to aeroallergens (data not shown).

Taken together, our study shows that both BNT162b2 and ChAdOx1 vaccines can effectively induce nasal NAbs, albeit variable in the ChAdOx1 arm. Why only some subjects develop local NAbs after vaccination in the former group is currently unclear and warrants follow up to fully understand the underlying immunological mechanisms. As it is unclear whether other local immunological mechanisms might induce protection against infection and viral shedding, induction of mucosal NAbs might be of key importance. Longitudinal follow-up of the described subjects is needed to see whether vaccines can induce long lasting neutralizing responses in the nasal mucosa. Failure to induce long-lasting NAbs warrants rational booster design or other strategies, such as nasal vaccination. Based on our findings, we advocate for the inclusion of nasal mucosa NAbs measurements in vaccine efficacy trials and routine testing procedures.

\begin{tabular}{lll}
\hline & BNT162b2 & ChAdOx1 \\
& No $(\%)$ & No $(\%)$ \\
$\mathbf{n}=$ & 24 & 22 \\
Sex & & \\
Female & $18(75 \%)$ & $18(82 \%)$ \\
Male & $6(25 \%)$ & $4(18 \%)$ \\
Age, median (IQR), y & $42.5(38.5-49.0)$ & $36.0(25.0-42.0)$ \\
BMI, median (IQR), kg/m2 & $25.0(23.0-27.1)$ & $23.7(20.6-26.5)$ \\
Current smoking & $1(4 \%)$ & $2(9 \%)$ \\
Prior COVID-19 infection & $12(50 \%)$ & $11(50 \%)$
\end{tabular}




\begin{tabular}{lll} 
Confirmed by RT-PCR & $9(75 \%)$ & $10(91 \%)$ \\
Confirmed by serology & $1(8 \%)$ & $1(9 \%)$ \\
$\begin{array}{l}\text { Self-reported } \\
\text { Patient reported allergy }\end{array}$ & $2(17 \%)$ & $0(0 \%)$ \\
Yes & $11(46 \%)$ & $8(36 \%)$ \\
No & $13(54 \%)$ & $14(64 \%)$ \\
\hline
\end{tabular}

Table I. Subject baseline characteristics.

\section{Hosted file} image1.emf available at https://authorea.com/users/396618/articles/530709-covid-19-
vaccination-with-bnt162b2-and-chadox1-vaccines-induces-nasal-neutralizing-antibodies

Figure I. Effect of vaccination on the induction of systemic and local neutralizing antibodies. A-B, Percentage of patients with SARS-CoV-2 neutralizing antibodies (a) and degree of ACE2 binding inhibition (b) in serum prior and post vaccination with BNT162b2 and ChAdOx1. C-D, Percentage of patients with SARS-CoV-2 NAbs (c) and degree of ACE2 binding inhibition (d) in nasal secretions prior and post vaccination with BNT162b2 and ChAdOx1.

Asterisks indicate statistical significance by two-way repeated-measures ANOVA followed by Sidak's multiple comparisons test. ${ }^{*}: \mathrm{p}<0.05,{ }^{* *}: \mathrm{p}<0.001,{ }^{* * *}: \mathrm{p}<0.0002,{ }^{* * * *}: \mathrm{p}<0,0001$.

\section{References}

1. Polack FP, Thomas SJ, Kitchin N, et al. Safety and Efficacy of the BNT162b2 mRNA Covid-19 Vaccine. NEJM. 2020; 383:2603-2615.

2. Voysey M, Costa Clemens SA, Madhi SA, et al. Safety and efficacy of the ChAdOx1 nCoV-19 vaccine (AZD1222) against SARS-CoV-2: an interim analysis of four randomized controlled trials in Brazil, South Africa, and the UK. The Lancet. 2021; 397:99-111.

3. Sungnak W, Huang N, Bécavin C, et al. SARS-CoV-2 entry factors are highly expressed in nasal epithelial cells together with innate immune genes. Nat Med. 2020; 26: 681-687.

4. Chan RWY, Liu S, Cheung JY, et al. Study on the mucosal and serological immune reponse to the novel coronavirus (SARS-CoV-2) vaccines. MedRxiv preprint doi: https://doi.org/10.1101/2021.06.15.21256661

5. Berings M, Arasi S, De Ruyck N, et al. Reliable mite-specific IgE testing in nasal secretions by means of allergen microarray. JACI. 2017; 140:301-303. 